\title{
Central Regions of AGNs Probed by the Neutral Hydrogen
}

\author{
Raffaella Morganti \\ Netherlands Foundation for Research in Astronomy, Postbus 2, \\ NL-7990 AA, Dwingeloo, NL
}

\begin{abstract}
The physical and kinematical conditions of the gas surrounding an active galactic nucleus (AGN) offer key diagnostics for understanding the processes occurring in the inner few kpc around the nucleus. Neutral hydrogen can give important insights on these regions. Apart from probing the presence of gas in relatively settled conditions (i.e. circumnuclear disks/tori) it can also trace the presence of extreme outflows. Some examples of these phenomena are briefly presented.
\end{abstract}

\section{Introduction}

The origin of activity in galaxies is often explained as triggered by merger and/or interaction processes. This idea is supported by morphological and kinematical evidence (e.g. Smith \& Heckman 1989, Tadhunter et al. 1989, Baum et al. 1992). Torques and shocks during the merger can remove angular momentum from the gas in the merging galaxies and this provides injection of substantial amounts of gas/dust into the central nuclear regions (see e.g. Mihos \& Hernquist 1996). It is, therefore, likely that in the initial phase of an AGN, this gas still surrounds (and possibly obscures) the central regions. AGN-driven outflows have powerful effects on this dense ISM. This feedback process could play an important part also in the evolution of the host galaxy as it may limit the growth of the super-massive black holes (BHs) and regulate the correlation between $\mathrm{BH}$ and bulge properties (see e.g. Silk \& Rees 1998).

Part of the gas will end up settling into a circumnuclear disk/torus. The presence of tori is an essential ingredient for the unified schemes for active galactic nuclei. Although it has been generally assumed that the tori are composed of dusty molecular clouds, it is now clear that, under certain conditions, they can be partly formed by atomic hydrogen (Maloney et al. 1996). Nuclear (optical) disks - with size ranging from 0.1 and $1 \mathrm{kpc}$ - are also detected in a large number of early-type galaxies (both radio-loud and radio-quiet). These disks (mainly detected by HST) can be seen either in ionized gas or through their strong dust absorption (van der Marel 2001, Capetti et al. 2000 and ref. therein).

All the above illustrates how important is the gas in the study of AGNs. As the gas is likely to be found in different phases (atomic, molecular and ionized), the study of all of these is crucial to obtain a complete view of the processes occurring. Neutral hydrogen is one of these components and in the nuclear regions of radio loud AGNs it has been very often detected in absorption against the stronger radio cores (see e.g. Heckman et al. 1983; van Gorkom et al. 1989; 


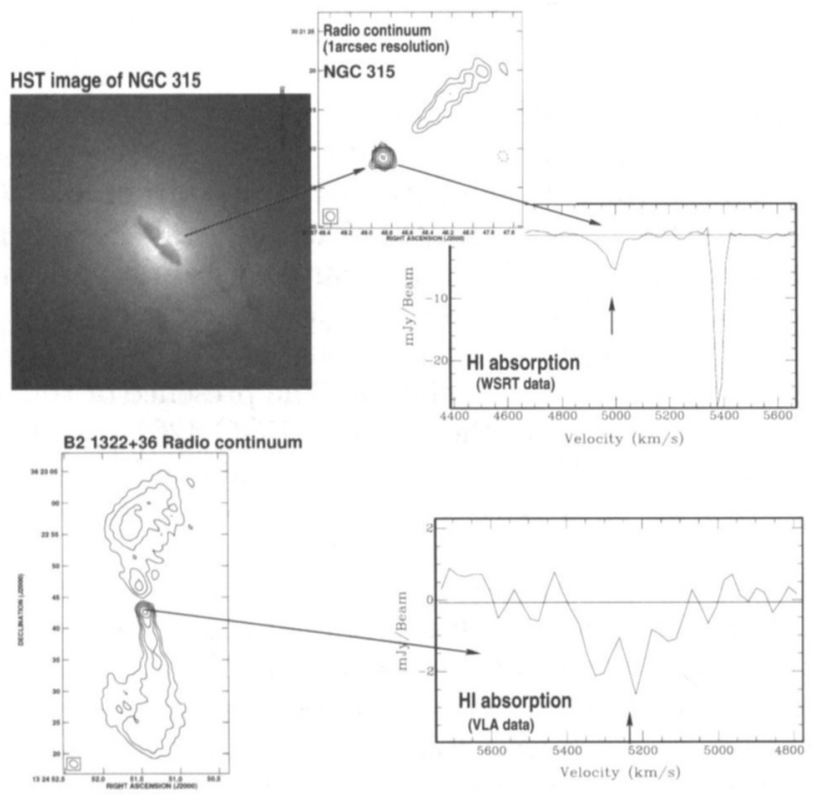

Figure 1. HI absorption detected with the WSRT and the VLA against the core of two radio galaxies: NGC 315 and B2 1322+36. The continuum images are also shown.

Morganti et al. 2001, 2002; Vermeulen et al. 2003). From the detection of the H I we can study: i) the gas that is settled in circumnuclear disks/tori. As highlighted above, this is relevant in the context of the unified schemes for AGNs; ii) the unsettled gas that might represent the leftovers from the merger that triggered the activity. From its kinematics we can study whether it represents in-falling gas (e.g. feeding the AGN) or out-flowing gas (perhaps relevant for feedback effects). Here, I briefly discuss some of the most recent results in this area.

\section{Settled gas}

As described above, settled H I gas can be associated with circumnuclear disks and tori. In powerful (i.e. Fanaroff-Riley II) radio galaxies, evidence for $\mathrm{HI}$ associated with tori has been found, e.g., in the case of the Cygnus A. In this object, a 50 pc-scale, rotating, flattened structure has been found from the VLBI observations (Conway 1999). Due to the weakness of the radio core in these type of galaxies, this study can be done only in very few cases. More promising is the study of $\mathrm{HI}$ absorption in powerful compact (steep spectrum) radio sources. These sources are uniquely suited for investigations into the physics of the central engines, in particular to study the kinematics of the gas within $100 \mathrm{pc}$ of the core (see e.g. Vermeulen et al. 2003). Evidence of H I associated with circumnuclear 
tori has been reported for some of these compact sources (see e.g. Conway 1997, Peck \& Taylor 2001) with thickness of about $100 \mathrm{pc}$ and column density of the order of $10^{23} \mathrm{~cm}^{-2}$ (with $\mathrm{T}_{\text {spin }}$ of several thousand $\mathrm{K}$ ). The information derived from the $\mathrm{HI}$ can be particularly useful to constrain characteristics the central torus when combined with hard X-ray data. This has been done in the case of two possible Compton-thick galaxies studied by Risaliti et al. (2003).

In low luminosity radio galaxies the situation could be different. The high detection rate of optical cores, the lack of large absorption in X-ray (Chiaberge et al. 1999) and possibly also the relatively low detection rate of $\mathrm{HI}$ absorption (Morganti et al. 2001) suggest that the standard pc-scale geometrically thick torus is not present in these radio galaxies. The presence of thin disks has been claimed from $\mathrm{H}$ I observations in the case e.g. NGC 4261 (van Langevelde et al. 2000). For this object, the VLBI data suggest that the H I absorption is due to a disk of only $\sim 1.3$ pe thick projected against the counter-jet. In NGC 4261, evidence for the presence of such nuclear disk are also found in HST images. The idea of thin disks can be investigated in more detail by correlating the presence (or absence) of $H I$ absorption with the optical characteristics. This has been done for a sample of radio galaxies (selected from Capetti et al. 2000) for which information about the presence of optical cores and nuclear dusty disks/lanes (from HST images) is available. Fig. 1 shows two examples of the detected objects. Interestingly, H I absorption was detected in the two galaxies that have dust disks/lanes and no optical cores. In these cases, the column density of the absorption is quite high $\left(>10^{21} \mathrm{~cm}^{-2}\right.$ for $\left.\mathrm{T}_{\text {spin }}=100 \mathrm{~K}\right)$ and the derived optical extinction $A_{B}$ (between 1 and 2 magnitudes) is such that it can, indeed, produce the obscuration of the optical cores. In the other two cases, H I absorption has been detected despite the presence of optical cores. However, the column density derived from the detected absorption is much lower $\left(\sim 10^{20} \mathrm{~cm}^{-2}\right.$ for $T_{\text {spin }}=100$ $\mathrm{K}$ ) and the derived extinction is of the order of only a fraction of a magnitude. This is, therefore, consistent with what expected if the circumnuclear disk are thin in these radio galaxies.

\section{Unsettled gas}

\subsection{Any evidence for infall?}

Evidence for in-falling gas was reported by van Gorkom et al. (1989). In a sample of radio galaxies, H I absorption was detected either close to the systemic velocity or systematically red-shifted, indicating therefore a prevalence of gas falling into the nucleus. This result does not appear to be confirmed by more recent observations. For example, the study of $\mathrm{HI}$ absorption in compact radio sources by Vermeulen et al. (2003) shows that there is evidence for significant gas motions and not only positive but even more negative $\mathrm{HI}$ velocities (up to more than $1000 \mathrm{~km} \mathrm{~s}^{-1}$ compared to the systemic velocity) are found. This is indicating that gas flowing out of the galaxy is also present. Indeed, clear cases of fast gas outflows have now been detected as described below.

One of the most promising case of in-falling gas was found in the radio galaxy NGC 315. A very narrow and highly red-shifted $\left(\sim 500 \mathrm{~km} \mathrm{~s}^{-1}\right) \mathrm{HI}$ absorption (see also Fig. 1) was reported by Heckman et al. (1983) and Dressel et al. (1983). VLBI observations (Peck 1999, Morganti et al. 2002) are now 

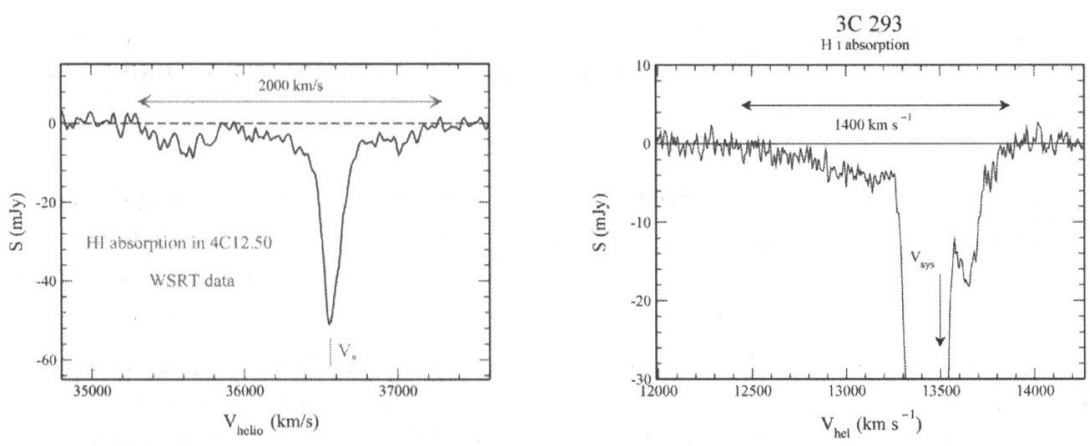

Figure 2. The H I absorption profile detected in 4C 12.50 (left) and 3C 293 (right) from the WSRT observations. The spectra are plotted in flux (mJy) against optical heliocentric velocity in $\mathrm{km} \mathrm{s}^{-1}$.

showing that this absorption appears to cover a region of about $9 \mathrm{pc}$ of the source, from the core to the first part of the jet. A likely explanation for this absorption is that of a cloud at large distance from the nucleus (like tidal debris) detected, in projection, against the nucleus (Morganti et al. 2002). This seems to be more favorable over the possibility of a small cloud falling into the nucleus and feeding the AGN.

\subsection{Outflows}

Fast nuclear outflows of ionized gas appear to be a relatively common phenomena in AGNs. It is, therefore, not too surprising to find such outflows also in radio galaxies (see Morganti et al. 2003a for a summary of recent results). However, it is extremely intriguing the discovery of a number of radio galaxies where the presence of fast outflows is associated not only with ionized but also with neutral gas. This finding gives new and important insights on the physical conditions of the gaseous medium around an AGN. The best examples so far are the radio galaxies 3C 293 (Morganti et al. 2003b) and 4C 12.50 and the Seyfert galaxy IC 5063 (Oosterloo et al. 2000). It is also worth noticing that outflows of ionized gas are also associated with these neutral outflows (see Morganti et al. 2003a). A number of possible hypotheses can be made about the origin of the gas outflow (e.g., star-burst winds, radiation pressure from the AGN adiabatically expanded broad emission line clouds) although at the moment the hypothesis that they are driven by the interaction of the radio jet with the ISM is favored. To investigate whether this is indeed correct, high-resolution (VLBI) studies are in progress to find the exact location of the out-flowing gas. So far these outflows have been found in objects that are either in the early-stage of their evolution (like $4 \mathrm{C} 12.50$ ) or, perhaps, in a phase of re-started activity (as might be the case for 3C 293). Another characteristic of these galaxies is the presence of a young stellar population (from their optical spectra, see e.g. Tadhunter et al. 2002). Such a component (with ages between 0.5 and 2 Gyr) can be considered an indication that the galaxy is indeed in a particular stage of its evolution, when large amounts of gas/dust - likely from the merger that triggered the activity - are still present in the inner region and the radio jet is strongly interacting 
with it. A more systematic search for fast gas outflows in radio galaxies is now in progress (with the WSRT) and has already revealed more cases of broad, blue-shifted H I absorptions.

\section{References}

Baum S. et al. 1992, ApJ 389, 208

Capetti et al. 2000 A\&A 362, 871

Chiaberge, M., Capetti, A., \& Celotti, A., 1999, A\&A, 349, 77

Conway, J.E. 1999 in Highly redshifted radio lines, Carilli C.L. et al. eds., ASP Conf. Series 156,259

Conway, J.E. 1997, in 2nd Workshop on GPS and CSS Radio Sources, Snellen et al. M.N. Publ JIVE, Leiden p.198

Dressel et al. 1983, ApJL 266, L97

Heckman et al. 1983, AJ, 88, 583

Maloney, P.R., Hollenbach D.J., \& Tielens A.G.G.M. 1996, ApJ 466, 561

Mihos, J.C. \& Hernquist L. 1996 ApJ, 464, 641

Morganti, R. 2002, in Issues in Unification of Active Galactic Nuclei, R. Maiolino et al. eds., ASP Conference Proceedings, Vol. 258 p.63 (astro-ph/0109056)

Morganti et al. 2003a, in Recycling Intergalactic and Interstellar Matter, IAU Symposium 217, eds. P.-A. Duc et al., in press (astro-ph/0310629)

Morganti, R., Oosterloo, T.A., Emonts, B.H.C., van der Hulst, J.M., \& Tadhunter, C.N. 2003b, ApJLetter 593, L69

Morganti, R. et al. 2002, 6th European VLBI Network Symposium, Ros E. et al. (Eds.), MPIfR, Bonn, Germany p.171

Morganti, R. et al. 2001, MNRAS 323, 331

Oosterloo, T.A. et al. 2000, AJ 119, 2085

Peck, A.B. \& Taylor G.B. 2001, ApJ 554, L147

Peck, A.B. 1999, PhD Thesis, New Mexico Inst. of Mining and Technology

Risaliti, G., Woltjer, L., \& Salvati, M. 2003 A\&A 401, 895

Silk, J. \& Rees, M.J. 1998, A\&A 331, L1

Smith \& Heckman 1989, ApJ 341, 658

Tadhunter, C., Fosbury \& Quinn P. 1989, MNRAS 240, 225

Tadhunter, C.N. et al. 2002, MNRAS 330, 977

van Gorkom, J.H. et al. 1989, AJ 97, 708

van Langevelde, H.J., Pihlström, Y.M., Conway J.E., Jaffe W., \& Schilizzi, R.T. 2000, A\&A 354,45

van der Marel, R. 2001, in 'Galaxies and their Constituents at the Highest Angular Resolutions' IAU Symp. 205, Schilizzi R. et al. eds., ASP, p.154

Vermeulen et al. 2003, A\&A 404, 861 